\title{
Ultrasound for the Detection of Pleural Effusions and Guidance of the Thoracentesis Procedure
}

\author{
Kamila Sikora, ${ }^{1}$ Phillips Perera, ${ }^{2}$ Thomas Mailhot, ${ }^{1}$ and Diku Mandavia ${ }^{1}$ \\ ${ }^{1}$ Department of Emergency Medicine, USC Medical Center, General Hospital, 1200 State Street, Los Angeles, CA 90033, USA \\ ${ }^{2}$ Division of Emergency Medicine, Stanford University Medical Center, 300 Pasteur Drive, Alway Building, M121, \\ Stanford, CA 94305, USA \\ Correspondence should be addressed to Phillips Perera, pperera1@mac.com
}

Received 5 August 2012; Accepted 23 August 2012

Academic Editors: R. Breitkreutz and C. C. Chang

Copyright () 2012 Kamila Sikora et al. This is an open access article distributed under the Creative Commons Attribution License, which permits unrestricted use, distribution, and reproduction in any medium, provided the original work is properly cited.

Objective. To review the use of ultrasound for the detection of pleural effusions and guidance of the thoracentesis procedure. Methods. Two clinical cases will be presented in which ultrasound proved beneficial in guiding the diagnosis and management of patients with pleural effusions and respiratory distress. The ultrasound techniques for the evaluation of pleural effusions and performance of the thoracentesis procedure are discussed. A review of the most current literature follows to present the known diagnostic and safety benefits of ultrasound guidance for thoracentesis. Conclusions. Ultrasound improves the diagnostic accuracy for the detection of pleural effusions over standard chest radiographs. Ultrasound can also diagnose a complicated pleural effusion that may be at higher risk for an adverse outcome during a thoracentesis. Optimally, thoracentesis should be performed under direct ultrasound guidance to decrease the complication rate and improve patient safety.

\section{Introduction}

Emergency and critical care patients in respiratory distress often require emergent interventions, including the immediate treatment of pleural effusions. Chest radiographs have traditionally served as the reference imaging component for determining the correct diagnosis and optimal management course of these patients. However, when specifically examining for the detection of pleural effusions, chest radiographs have been found to be relatively less sensitive than ultrasound. On an upright posterior-anterior projection chest radiograph, pleural effusions are generally recognized to become visible as blunting of the lateral costophrenic angle at a volume of $150-200 \mathrm{cc}$. Obtaining an upright lateral chest radiograph can further improve the detection of pleural effusions. On this view, $50 \mathrm{cc}$ of fluid is the recognized volume that can be seen as blunting of the posterior costophrenic angle [1-3]. In contrast, ultrasound has been demonstrated to detect as little as $20 \mathrm{cc}$ of pleural fluid $[2,4]$. One study directly compared chest radiography to ultrasound for the diagnosis of pleural effusion, with the gold standard being computed tomography of the chest.
Chest radiography had a sensitivity of $65 \%$, a specificity of $81 \%$, and diagnostic accuracy of $69 \%$, while ultrasound had a sensitivity of $100 \%$, a specificity of $100 \%$, and a diagnostic accuracy of $100 \%$ [5].

To directly compare the accuracy of chest radiographs to ultrasound for detection of pleural effusions amenable to thoracentesis, one study enrolled 40 patients who underwent a total of 45 thoracentesis procedures, with the gold standard being the successful removal of fluid. Ultrasound was able to diagnose pleural effusions in 17 of the 45 patients where the fluid was not adequately visualized on chest radiography [6]. As compared to chest radiographs, ultrasound has been demonstrated to better quantify the amount of the effusion present in the chest cavity [7]. Furthermore, ultrasound can determine if a complicated pleural effusion is present, such as an effusion with fibrin stranding and loculations [8]. In one study in children, ultrasound performed with a similar accuracy to computed tomography in the detection of complicated pleural effusions [9]. These studies indicate that ultrasound is a better test than chest radiography for the diagnosis and characterization of pleural effusions. Some have suggested that due to this improved accuracy, the 


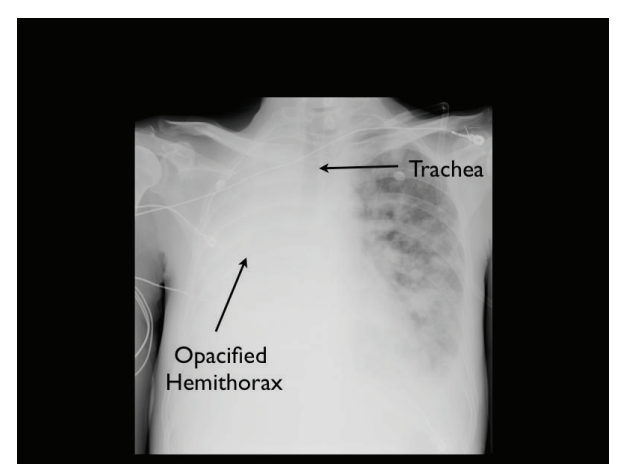

FIgure 1: Chest radiograph from patient in Case 1. Note the complete opacification of the right hemithorax. The trachea appears to be deviated toward the affected hemithorax.

use of ultrasound could potentially decrease the number of chest radiographs needed in critically ill patients [10]. Ultrasound has also been demonstrated to perform with a sensitivity similar to chest computed tomography scan for this indication. Thus, the current literature confirms that bedside ultrasound is a rapid and accurate means to help decide if a clinically significant pleural effusion is present for which a thoracentesis might be indicated.

\section{Report of Cases}

2.1. Case 1. A 50-year-old female with a history of lung cancer presented to the Emergency Department with shortness of breath for one day. Her vital signs included a blood pressure of $98 / 68$, a heart rate of 144 beats per minute, a respiratory rate of 28 breaths per minute, a temperature of $100.5 \mathrm{~F}$, and a pulse oxygenation of $90 \%$ on room air. On arrival, she was immediately placed on supplemental oxygen with improvement in the oxygen saturation to $94 \%$. On physical examination, the patient had poor air movement bilaterally with decreased breath sounds on the right side. A portable chest radiograph demonstrated complete opacification of her right hemithorax with tracheal deviation toward the right. Multiple infiltrative lesions were also noted in the left lung (Figure 1).

The critical clinical questions for the treating physicians were first, how to arrive at the correct diagnosis with this lung pathology? Did the right lung opacification represent a pleural effusion or a mass? Second, what was the optimal treatment plan for the patient? Would a thoracentesis procedure be indicated in this case to help alleviate respiratory distress? To answer these questions, an ultrasound machine was brought directly to the bedside. Imaging of the right and left lungs was performed using both a lower frequency $3 \mathrm{MHz}$ and a higher frequency $10 \mathrm{MHz}$ probe. Using ultrasound, the right lung was noted to have complete consolidation and appeared as a solid mass. No pleural effusion was noted on the right side (Figures 2 and 3). It was thought that this consolidation was due to invasion and atelectasis secondary to her known primary lung adenocarcinoma. A prior computed tomography scan image of the right chest is shown as reference (Figure 4). Interestingly, ultrasound of

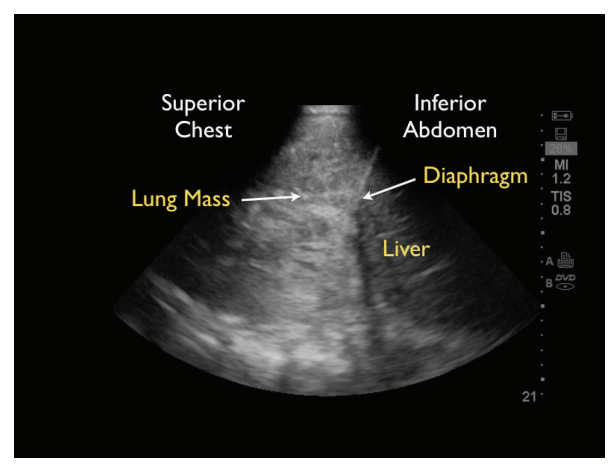

FIGURE 2: Ultrasound of the right hemithorax obtained from the patient in Case 1. This ultrasound was taken with a $3 \mathrm{MHz}$ probe. Note consolidation of the lung, visualized as a mass superior to the diaphragm.

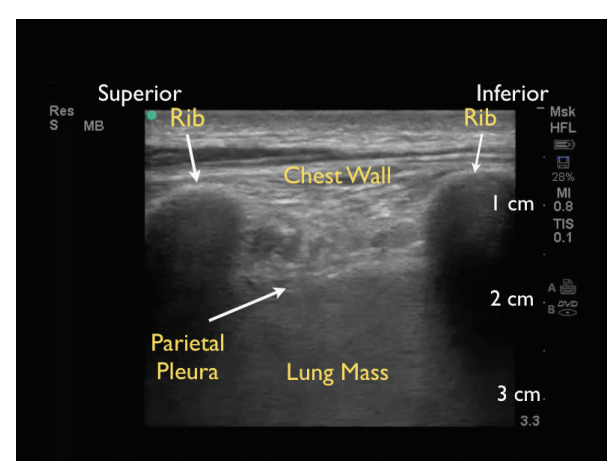

FIGURE 3: Ultrasound of the right hemithorax obtained from patient in Case 1. This ultrasound was taken with a $10 \mathrm{MHz}$ probe. Note complete consolidation of the lung, which is visualized as a mass deep to the parietal pleura.

the left thorax showed a small pleural effusion that was not appreciated on chest radiography (Figure 5). A more detailed ultrasound examination also confirmed that there was no pericardial effusion and that the patient had hyperdynamic cardiac motion.

Her laboratory tests were significant for an elevated white blood cell count of 17,000. Based on the presence of both the chest radiograph and ultrasound findings, in addition to the fever and the elevated white blood cell count, the patient was diagnosed with pneumonia. Broad spectrum intravenous antibiotics were initiated. Thoracentesis of the right thoracic cavity was not performed based on the absence of fluid on ultrasound examination. Drainage of the left thoracic cavity was also deferred at that time, as during a full respiratory cycle the lung was noted to move into an intervening position in front of the fluid. This lung position in relation to the effusion would have increased the potential complication rate of a thoracentesis. The patient was then admitted to the hospital for further supportive management.

2.2. Case 2. A 42-year-old female with a history of breast cancer currently under chemotherapy presented to the Emergency Department with increasing shortness of breath 


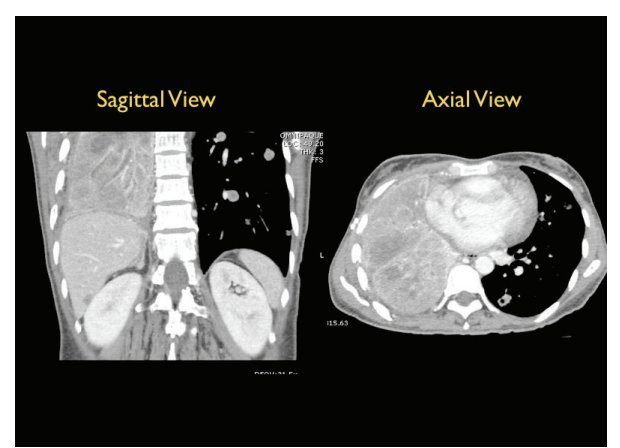

FIgURE 4: Chest computed tomography scan obtained from patient in Case 1. Note the mass-like appearance of the right lung in both axial and sagittal views.

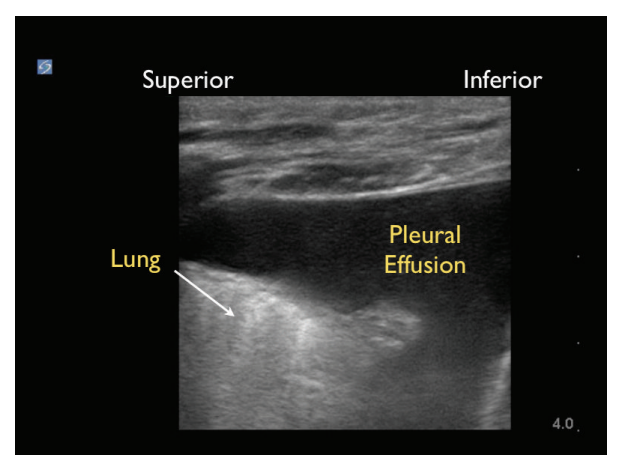

FIGURE 5: Ultrasound of the left hemithorax obtained from patient in Case 1. This ultrasound was taken with a $10 \mathrm{MHz}$ probe. A small pleural effusion, appearing dark or anechoic and located anterior to the lung, is noted on this view.

for two days. On physical examination, the patient appeared to be in severe respiratory distress when she attempted to lie flat. Her breathing improved moderately when she sat directly upright. Her vital signs included a blood pressure of $130 / 92$, a heart rate of 110 beats per minute, a respiratory rate of 30 breaths per minute, a temperature of $99.6 \mathrm{~F}$, and a pulse oximetry of $91 \%$ on room air. A portable chest radiograph was obtained (Figure 6). Complete opacification of the left lung is noted with tracheal deviation away from the affected hemithorax to the right.

Given the patient's respiratory distress, a thoracentesis procedure was considered to drain the presumed large left pleural effusion. An ultrasound machine was brought to the bedside and images were obtained using both the $3 \mathrm{MHz}$ and $10 \mathrm{MHz}$ probes (Figures 7 and 8). A large left side pleural effusion was immediately diagnosed and the patient was offered a therapeutic thoracentesis. After obtaining informed consent, a thoracentesis was then performed under direct ultrasound guidance and 1 liter of serosanguineous fluid was removed and sent to the laboratory for analysis. Immediately following the procedure, the patient felt much improved and was able to breathe more comfortably in a semirecumbent resting position. She was then admitted to the hospital for further care after a follow-up chest radiograph showed

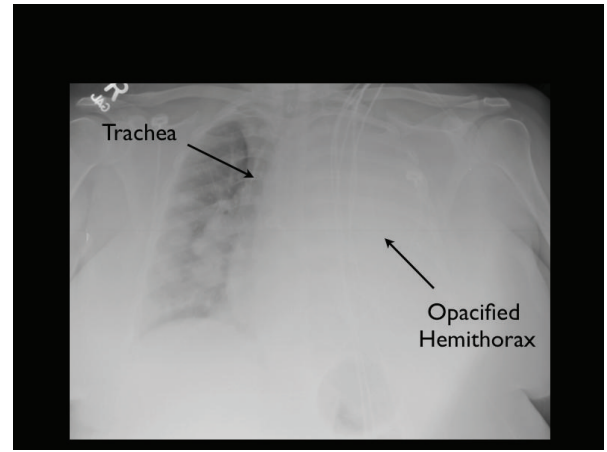

FIgure 6: Chest radiograph from patient in Case 2. Note the complete opacification of the left hemithorax. The trachea appears to be deviated away from the affected hemithorax.

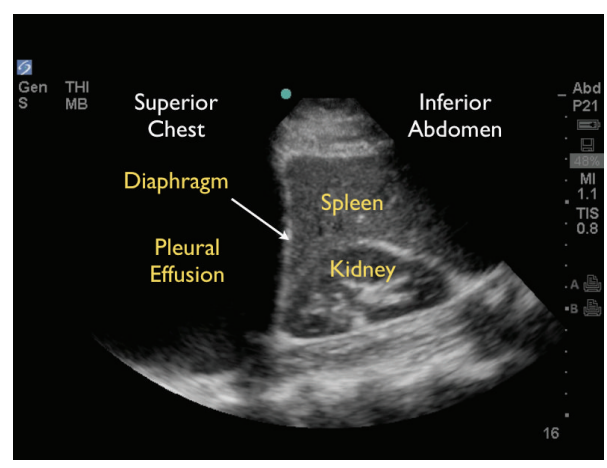

FIGURE 7: Ultrasound of the left hemithorax obtained from patient in Case 2. This ultrasound was taken with a $3 \mathrm{MHz}$ probe. Note the presence of a large pleural effusion, appearing as a dark or anechoic fluid collection, superior to the diaphragm.

substantial decrease in the size of the effusion and the absence of any iatrogenic pneumothorax.

These clinical cases demonstrate how ultrasound was extremely helpful in evaluating these patients with two very different etiologies (mass versus effusion) for the opacification of the hemithorax on chest radiography. M-mode Doppler can also be very useful in discriminating a pleural effusion from a lung mass (Figure 9). Finally, ultrasound also aided the clinicians in performing the thoracentesis procedure safely and without complication in the patient discussed in Case 2.

\section{Indications for Chest Ultrasound: Evaluation of Pleural Effusions}

Many older sources recommend initial use of the $3-5 \mathrm{MHz}$ curvilinear or phased array probe to view the pleural effusion and the surrounding landmarks. These include visualizing the lung within the pleural effusion, the diaphragm, the liver on the right side, and the spleen on the left side. Use of the lower frequency $3-5 \mathrm{MHz}$ probe offers a wider depth of field and a more global view of the lung and the effusion. Some publications find this single approach to be sufficient [11]. 


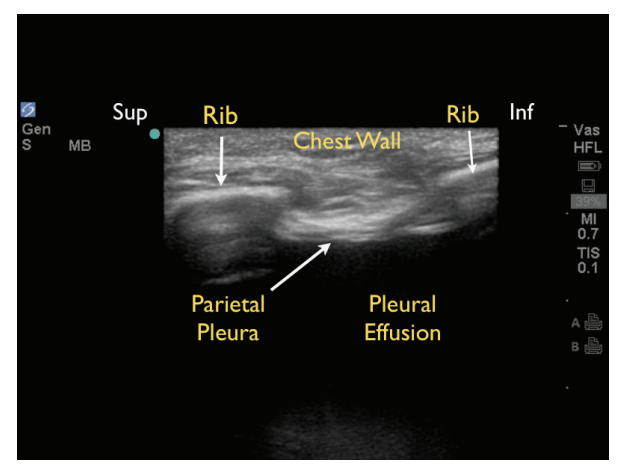

FIgURE 8: Ultrasound of the left hemithorax obtained from patient in Case 2. This ultrasound was taken with a $10 \mathrm{MHz}$ probe. Note the presence of a large pleural effusion, appearing as a dark or anechoic fluid collection, deep to the parietal pleura.

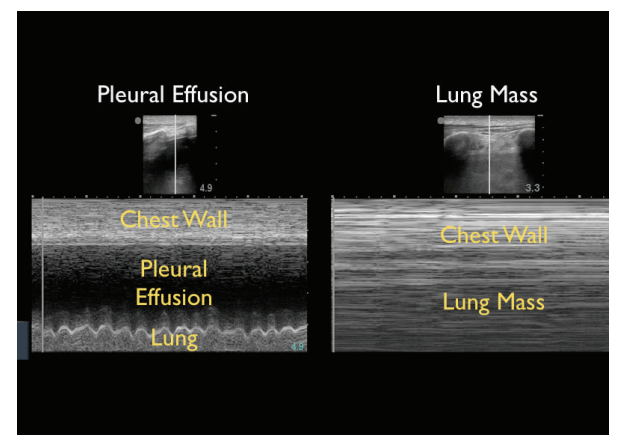

Figure 9: M-mode doppler ultrasound demonstrating discrimination of a pleural effusion (left side) from a lung mass (right side). This ultrasound was taken with a $10 \mathrm{MHz}$ probe. On the left, note the presence of the pleural effusion, appearing as a dark or anechoic fluid collection, with the lung seen moving in the background. On the right side, note the linear repeating lines of both the chest wall and the lung indicating an absence of motion of the lung due to the presence of the solid mass.

A more focused and detailed view of the thoracic space can then be obtained by using the higher frequency $10 \mathrm{MHz}$ linear array probe that allows improved imaging at a more superficial depth [12]. This technique can often allow one to more precisely measure the depth of the effusion from the outer parietal chest pleura to the lung. One can refer to this fluid depth as the safety zone, in which the needle should be positioned during the procedure. The clinician can also use the higher frequency probe to determine the exact position of the rib space that would be best for needle placement.

Ultrasound can be used to accurately estimate the amount of effusion present in the chest cavity and to decide how much fluid should optimally be removed during the thoracentesis procedure. One study described a method for estimating the size of the pleural effusion in medical intensive care unit patients. In this study, an equation was described between the volume of the effusion and the separation distance between the lung and the outer parietal pleura as

the effusion size $(\mathrm{cc})=20 \times$ separation $(\mathrm{sep})$ in $\mathrm{mm}$,

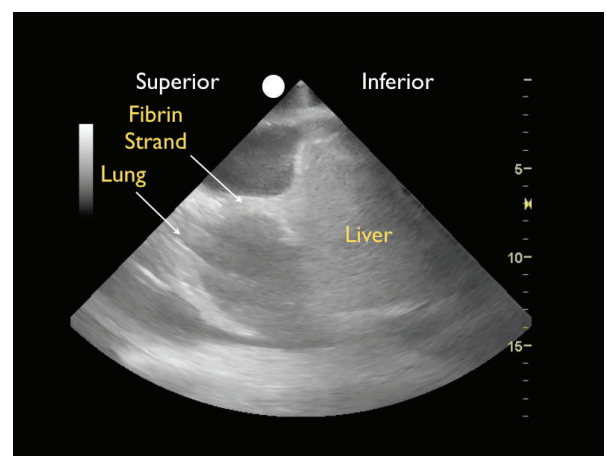

FIgURE 10: Ultrasound of a loculated pleural effusion. This ultrasound was taken with a $3 \mathrm{MHz}$ probe. Note the presence of a fibrin strands extending from the lung to the diaphragm, resulting in loculations of the pleural effusion.

where the separation (sep) was identified as the maximal separation distance between the parietal and visceral pleura during end-expiration. In this study, a lower frequency probe was used [13].

In pediatric patients, ultrasound of pleural effusions can also be very helpful. One comprehensive review of chest ultrasonography in pediatric patients noted that a significant proportion of children may have very small pleural effusions noted on ultrasound. A key difference in children is that their cartilaginous ribs are more easily penetrated with ultrasound, allowing improved thoracic ultrasound imaging. Thus, small pleural effusions in children may be more easily detected on bedside ultrasound. Ultrasound has also been used to successfully detect complicated pleural effusions in children [14].

\section{Thoracentesis Techniques}

Thoracentesis was long performed with only chest radiography and clinical exam as guidelines. Unfortunately, there was a significant morbidity associated with the procedure, chiefly the risk of pneumothorax [1]. Integrating ultrasound guidance into the procedure offers a significant safety benefit; however, it is crucial to know how best to use this imaging technique to achieve optimal outcomes.

The first step is to perform a proper assessment of the thoracic cavities to inspect for the presence of a pleural effusion. Most effusions will have a dark, or anechoic, appearance on ultrasound. Complicated pleural effusions may have a different appearance. Fresh blood in the pleural space will result in a brighter, or more hyperechoic, appearance [8]. Pleural empyemas may be recognized by the presence of gas bubbles within the fluid. The presence of fibrin stranding leading to lung adhesions or loculations can also be determined with ultrasound (Figure 10). The presence of septations from inflammatory stranding has been correlated with an increased need for surgical drainage in children [15]. Unfortunately, the presence of complex septations within a pleural effusion has also been associated with a poorer outcome for successful catheter drainage and a higher overall patient mortality rate (Figure 11) [16, 17]. 


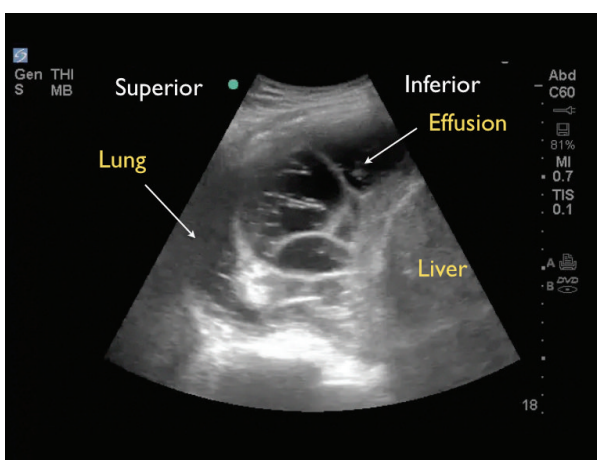

FIgURE 11: Ultrasound of a septated pleural effusion. This ultrasound was taken with a $3 \mathrm{MHz}$ probe. Note the presence multiple strands forming a septated pleural effusion.

Color flow Doppler imaging may be helpful in differentiating an empyema from the more vascular lung abscess [18].

Once a pleural effusion has been diagnosed, the second step is to determine if there is enough fluid to be safely removed. The British Thoracic Society recommends that pleural effusions amenable to safe thoracentesis have at least a $10 \mathrm{~mm}$ depth, resulting in a sufficient separation of the visceral lung pleura from the outer thoracic cavity parietal pleura. Once the patient is best positioned for the procedure, one can then use ultrasound to determine the optimal needle trajectory to the effusion. There should also be the absence of any intervening lung on the planned approach to the effusion. The movement of the lung should be viewed through a maximal respiratory cycle. Finally, the location of the diaphragm should be determined, in order to avoid this structure with the needle [19]. In a mechanically ventilated patients, a pleural fluid depth of at least $15 \mathrm{~mm}$ visualized over three intercostal spaces has been shown to be associated with a safer thoracentesis [6].

As mentioned above, using a combination of both the $3 \mathrm{MHz}$ and $10 \mathrm{MHz}$ probes can offer complementary views of the pleural effusion and thoracic cavity before and during a thoracentesis procedure. There are two general techniques for integrating ultrasound guidance into the thoracentesis procedure. The first is the static technique. In this technique, ultrasound is used to mark the optimal needle placement after the patient has been positioned for the procedure. This is referred to as the static technique, as the needle is not visualized throughout the procedure. Alternatively, one can place the probe in a sterile sheath and perform the procedure using the real-time, or dynamic, technique. This is the technique favored for procedures, like central line placement under ultrasound guidance. There is some controversy over which technique (static versus dynamic) is best for guidance of the thoracentesis procedure. However, some clinicians, including the authors, prefer the additional guidance of the dynamic technique, which allows one to image the needle as it enters the thoracic cavity and the effusion. The resultant real time visualization of the needle in relation to the lung as it moves with respirations can potentially allow one to best avoid accidentally puncturing this structure.

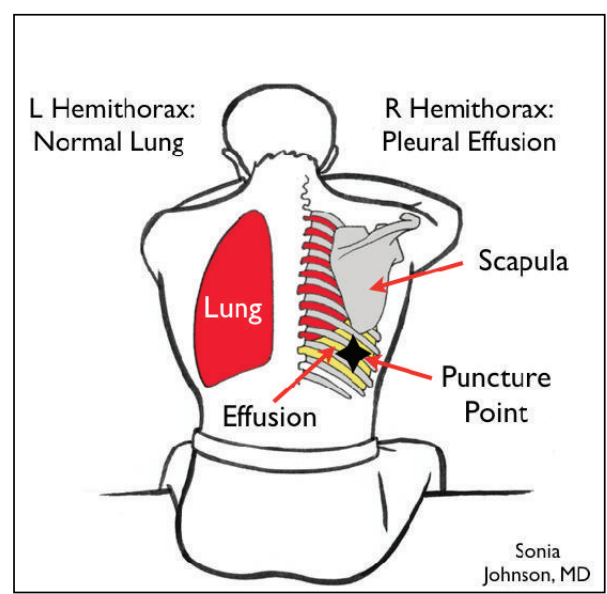

FIgure 12: Patient positioning for the posterior thoracentesis approach. Note that the patient should be sitting forward over a support. The location of needle placement is best determined by using ultrasound. A typical puncture point is shown in this illustration.

Prior to any invasive procedure like a thoracentesis, informed consent should be obtained from the patient and a time out procedure observed. The hemithorax identified for the procedure should be reconfirmed. The overlying skin area should then be prepared and draped in a sterile manner. The practitioner should also practice maximal sterile precautions throughout the procedure. After thorough hand washing, a mask, a sterile cap, gown, and gloves should be used. There are 2 options for positioning of the patient for the thoracentesis procedure. An upright position is traditionally preferred by many clinicians, with the patient leaning forward on a support. This allows access to the posterior approach to thoracentesis (Figure 12) [20]. The supine position, allowing a lateral approach to the chest cavity, may be employed in patients unable to sit up. This would be a similar position to that used for the typical placement of a chest tube (Figure 13). Optimally, the head of the bed should be elevated in this position to facilitate the drainage of the pleural fluid inferiorly and to accumulate the effusion closer to the location of standard needle placement [21].

Once the optimal needle puncture location is determined by using ultrasound, it is important to use a liberal amount of local anesthesia to provide maximal patient comfort during the procedure. Anesthesia should be infiltrated down to the parietal pleura, as this is the location where many nerve fibers are concentrated and patients often experience maximal pain. Dynamic ultrasound guidance can be used to guide the anesthetic needle down to the pleura and to avoid the intercostal vascular structures. It is critical to advance the needle over the top of the rib and to avoid the neurovascular bundle, which lies directly below the rib (Figure 14).

For a simple diagnostic thoracentesis, a 20 gauge needle and syringe is generally sufficient for fluid collection. For greater volume drainage, a specific thoracentesis kit has the benefit of a plastic catheter that can be advanced over a metal trocar. Removing the metal trocar immediately as the pleural 


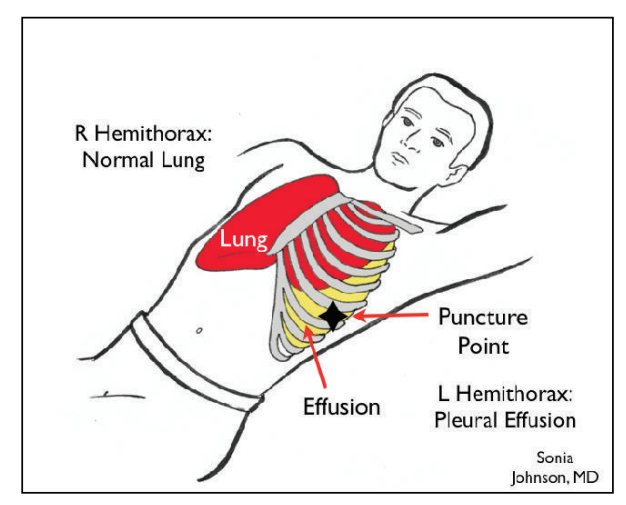

FIGURE 13: Patient positioning for the lateral thoracentesis approach. Note that the patient should be lying supine with the arm extended and the head of the bed elevated. The location of needle placement is best determined by using ultrasound. A typical puncture point is shown in this illustration.

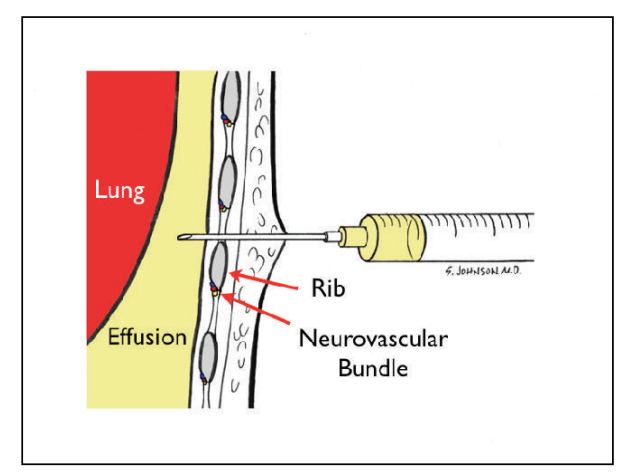

FIGURE 14: Needle trajectory should be positioned above the rib. Note that the neurovascular bundle is located inferior to the rib and must be avoided during the thoracentesis procedure.

effusion is entered, while simultaneously advancing the flexible plastic catheter, will have the benefit of decreasing the risk of injury to the lung. When ready to proceed, one should advance the needle into the pleural space while simultaneously drawing back with the plunger of the syringe. Once pleural fluid is flowing into the syringe, adequate fluid (usually $20-30 \mathrm{cc}$ ) should be removed and sent for the usual laboratory studies. These should include a cell count, glucose, LDH, fluid ph (sent separately in an arterial blood gas syringe) and a gram stain and culture. As much fluid as possible should be sent for the fluid culture, as bacteria in the pleural space may be relatively dilute. It is important to keep the needle and aspiration system sealed at all times and to avoid accidental inflow of air into the thoracic cavity, which could cause an iatrogenic pneumothorax.

After the thoracentesis procedure is complete and the needle or catheter is removed, standard practice currently dictates evaluation for a pneumothorax with a chest radiograph. However, one study offered a different view. In a review of 434 cases of ultrasound-guided thoracentesis, 10 cases of postprocedure pneumothorax were included.
In these cases of pneumothorax, only 2 of the intubated patients and none of the nonintubated patients required chest tubes. The authors suggest that in awake, nonintubated patients without respiratory distress following thoracentesis, a postprocedure chest radiograph is not necessary, as no resulting pneumothoraces were clinically significant [22]. Since that study, another paper recommended checking with ultrasound for pneumothoraces before and after the procedure, as an alternative to chest radiography [23].

\section{Complications of Thoracentesis}

Similar to any procedure, thoracentesis even under ultrasound guidance is associated with potential complications. In 2011, the results of an extensive literature review on the topic were published. This study identified multiple factors associated with increased patient morbidity, breaking them down into specific categories. Intrinsic patientrelated factors included the presence of smaller effusions $<250 \mathrm{cc}$, multiloculated effusions, underlying coagulopathy, obesity, poor patient positioning, mechanical ventilation, and pleural visceral adhesions. Procedural factors included inexperienced practitioners, lack of ultrasound guidance, and large volume drainage ( $>1.5$ liter). Systemic factors included inadequate support staff, the lack of a standardized procedural practice guideline, and the lack of both quality metrics and physician-specific outcome measurements [24].

Other studies have evaluated the association of various types of pleural effusions with rates of drainage success and subsequent complications. One paper found that massive transudative pleural effusions and malignant pleural effusions were associated with the greatest success rate $(81.6 \%$ and $75.5 \%$, resp.) [45]. Conversely, the same research group found in another study that these massive transudative pleural effusions were also associated with the highest overall complication rates (6 of 33, or 18\%) [36].

More specifically, literature related to the specific complications of the thoracentesis procedure include the following.

\section{Pneumothorax}

6.1. Overall Incidence of Pneumothorax and Impact of Ultrasound. The most commonly reported severe complication of thoracentesis is iatrogenic pneumothorax. Our literature review found 23 studies from 1978 to 2010, which evaluated the rate of pneumothorax associated with the thoracentesis procedure (see Table 1). In those studies looking specifically at the rates of pneumothorax resulting as a complication of thoracentesis performed without ultrasound, the incidence rate ranged from $4.3 \%$ to $30 \%$ of cases. In contrast, the pneumothorax rate associated with ultrasound-guided thoracenteses was lower, ranging from 0 to $9.1 \%$. The rates of subsequent chest tube insertion for pneumothoraces ranged from $2.2-7 \%$ in those procedures without ultrasound guidance and $0-3.7 \%$ in procedures performed with ultrasound guidance $[6,13,22,25-44]$.

A comprehensive 2010 meta-analysis included in the above analysis pooled 24 studies that reported the incidence of pneumothoraces following 6,605 thoracentesis 
TABLE 1: Literature review comparing studies looking at the rates of pneumothorax associated with the thoracentesis procedure. Indexed to primary study author, rate of pneumothorax, and the rate of required chest tubes or Heimlich valves. Note columns corresponding to studies using ultrasound to the left and no ultrasound to the right. Prospective studies (Pro), retrospective studies (Retro), randomized controlled trials (RCT) and meta-analysis (MA) study types are all included in this review.

\begin{tabular}{|c|c|c|c|c|}
\hline \multirow{2}{*}{ Author } & \multicolumn{2}{|c|}{ Pneumothorax rate } & \multicolumn{2}{|c|}{ Chest tube/Heimlich rate } \\
\hline & Ultrasound guided & No ultrasound & Ultrasound guided & No ultrasound \\
\hline Adams and Galati 1978 Pro [25] & Not given & & Not given & \\
\hline Balik et al. 2006 Pro [13] & 0 of $92(0 \%)$ & & & \\
\hline Barnes et al. 2005 Retro [26] & 15 of $305(4.9 \%)$ & 15 of $145(10.3 \%)$ & 2 of $305(0.7 \%)$ & 6 of $145(4.1 \%)$ \\
\hline Boland et al. 1998 Retro [27] & 19 of $512(5.7 \%)$ & & & \\
\hline Brandstetter et al. 1994 Pro [28] & 28 of $106(26 \%)$ & & & \\
\hline Colt et al. 1999 Pro [29] & 14 of $255(5.1 \%)$ & & 2 of $255(0.8 \%)$ & \\
\hline Duncan et al. 2009 Pro [30] & 4 of $362(1.1 \%)$ & 5 of $58(8.7 \%)$ & 0 & \\
\hline Gervais et al. 1997 Pro [22] & 6 of $342(1.8 \%)$ & 4 of $92(4.3 \%)$ & 0 & 2 of $92(2.2 \%)$ \\
\hline Grogan et al. 1990 RCT [31] & 0 of $19(0 \%)$ & 10 of $33(30 \%)$ & & \\
\hline Harnsberger et al. 1983 [32] & 0 of $110(0 \%)$ & & & \\
\hline Heidecker et al. 2006 Retro [33] & 9 of $367(2.5 \%)$ & & & \\
\hline Josephson et al. 2009 Pro [34] & 34 of $735(4.6 \%)$ & & 11 of $735(1.5 \%)$ & \\
\hline Jones et al. 2003 Pro [35] & 24 of $941(2.6 \%)$ & & 8 of $941(0.9 \%)$ & \\
\hline Liang et al. 2009 Retro [36] & 0 of $133(0 \%)$ & & & \\
\hline Lichtenstein et al. 1999 Pro [6] & 0 of $45(0 \%)$ & & & \\
\hline Mayo et al. 2004 Pro [37] & 3 of $232(1.3 \%)$ & & 3 of $232(1.3 \%)$ & \\
\hline Mynarek et al. 2004 Retro [38] & 20 of $711(2.8 \%)$ & & 0 & \\
\hline O’Moore et al. 1987 Retro [39] & 17 of $187(9.1 \%)$ & & 7 of $187(3.7 \%)$ & \\
\hline Pihlajamaa et al. 2004 Retro [40] & 11 of $264(4.2 \%)$ & & $1(0.4 \%)$ & \\
\hline Raptopoulos et al. 1991 Retro [41] & 5 of $188(2.7 \%)$ & 27 of $154(17.5 \%)$ & $2 \%$ & $7 \%$ \\
\hline Ravin 1977 Retro [42] & 0 of $6(0 \%)$ & & & \\
\hline Yu et al. 1992 Pro [43] & 1 of $25(4 \%)$ & & & \\
\hline Gordon et al. $2010 \mathrm{MA}$ [44] & $4 \%$ & $9.3 \%$ & & \\
\hline
\end{tabular}

procedures. The overall pneumothorax rate was $6.0 \%$ and $34 \%$ of these patients subsequently required chest tube placement. Use of ultrasound for guidance of the procedure was associated with a significantly lower risk of pneumothorax, with an odds ratio of 0.3 [44]. In a study that examined the rate of pneumothorax before and after institution of a policy of ultrasound guidance for all procedures, the results were convincing. The baseline rate of pneumothorax had been $8.6 \%$ without ultrasound guidance. After institution of ultrasound guidance, the iatrogenic pneumothorax rate decreased to $0.7-1.3 \%$ [30]. Based on this prevailing literature, a recent review article urged the inclusion of ultrasound guidance for the thoracentesis procedure as "a best practice" technique [24]. In addition, the British Thoracic Society and a leading panel of international specialists both support this position in their guideline $[19,46]$.

6.2. Association with Interstitial Lung Disease and Chronic Obstructive Lung Disease. During a thoracentesis procedure, pneumothorax may occur due to inadvertent lung puncture. Interestingly, this complication has a higher incidence in patients with underlying lung disease, such as interstitial lung disease and chronic obstructive lung disease, conditions that result in decreased lung compliance. Potentially, these stiffer lungs have an increased risk for needle puncture and resulting pneumothorax. One study specifically investigated the association of post-thoracentesis pneumothoraces and lung findings on chest computed tomography scan. They found that the pneumothoraces that did not heal spontaneously were more likely to be associated with the presence of underlying parenchymal lung disease [27]. Other researchers used manometry during the thoracentesis procedure in patients with underlying lung disease to show that less compliant lungs, termed "unexpandable lungs," were increasingly associated with pneumothoraces. The authors hypothesized that nonuniform stress distribution across the visceral pleura of these lungs that developed during thoracentesis may have resulted in parenchymal-pleural fistulae and resulting pneumothoraces [33].

The presence of advanced chronic obstructive pulmonary disease (COPD) directly increases a patient's risk for the development of post-thoracentesis pneumothorax. One study of 106 patients undergoing non-ultrasoundguided thoracentesis found that $41.7 \%$ of those with COPD developed a pneumothorax, compared to only $18.5 \%$ of those without COPD [28].

6.3. Association with Prior Thoracenteses. One study investigated potential predictors for pneumothoraces and found the only statistical significant factor to be a history of a prior 
thoracentesis on the same hemithorax. These patients were found to have a pneumothorax rate of 5 in 40 procedures, or an incidence of $12.5 \%$ [29]. Clinicians might factor this risk into planning another procedure on any affected patient.

6.4. Association with Amount of Pleural Effusion Removed. One study specifically evaluated the risk of pneumothorax with the removal of different fluid volumes. In patients with $>1100 \mathrm{cc}$ fluid removed, there was a significantly increased incidence of pneumothorax [35]. A prospective observational study specifically evaluated the relationship between drained fluid volume and risk of pneumothorax. This study found an odds ratio of 3.8 when 1.8-2.2 liters was removed. The odds ratio increased to 5.7 with removal of 2.3 liters or more [34].

\section{Other Significant Complications of Thoracentesis}

7.1. Reexpansion Pulmonary Edema. Traditionally, it has been taught that large volume thoracentesis was increasingly associated with the complication of reexpansion pulmonary edema. However, one study mentioned above specifically looked at large volume thoracentesis and found that only $0.5 \%$ ( 2 of 373 ) of those patients with $>1$ liter of fluid removed actually developed this complication [35]. As previously emphasized, removal of large volumes may however be associated with higher rates of pneumothorax [34].

7.2. Abdominal Viscus Injury. Other potential serious complications include splenic and liver laceration, although little data currently exists on the rate of these complications. These could result from inadvertent placement of the needle below the diaphragm. This is why it is important to accurately determine the location of these structures with ultrasound prior to the procedure.

7.3. Infection Causing Empyema. Infection is another recognized potential adverse outcome. One study found that the rate of complicating infection associated with the placement of pigtail catheter drains occurred in 4 of 332 patients, or an incidence rate of $1.2 \%$ [45]. This demonstrates a low incidence of infection. However, strict adherence to sterile technique is advised through the procedure to decrease this complication.

7.4. Other Complications of Thoracentesis. In a prospective study of 941 thoracentesis procedures, researchers looked at the incidence of symptomatic reactions following the procedure. Complications included pain (25\%), shortness of breath $(1 \%)$, cough $(0.8 \%)$, and vagal reaction $(0.6 \%)$ [35].

\section{Conclusion}

Ultrasound serves as a more accurate imaging tool than chest radiography for the diagnosis of pleural effusions. Ultrasound has the additional potential benefits that it can be rapidly performed, repeated over time and lacks the ionizing radiation associated with both chest radiographs and computed tomography scans. As described in the patient cases above, bedside ultrasound can allow discrimination of pleural effusions from other lung pathology that may appear similar on a chest radiograph. Furthermore, ultrasound can allow diagnosis of complicated pleural effusions, such as empyemas and abscesses that may be associated with a higher risk for a drainage procedure.

Our literature review, as well as that performed by others, demonstrates a clear and convincing decrease in the overall complication rate associated with thoracentesis when ultrasound guidance is employed during the procedure. The most dramatic improvement was noted in the rate of pneumothorax, which is the most common recognized complication associated with the procedure. Specialty societies and expert consensus panels now urge integration of ultrasound into the thoracentesis procedure as a "best practice" guideline. In the age when improving patient safety is paramount to the care delivered in hospitals across the world, ultrasound has a proven benefit that fits in perfectly with the campaign to decrease procedural complications and the resulting patient morbidity and mortality. Physicians in the many specialties that perform the thoracentesis procedure should be urged to learn ultrasound and to use this application whenever possible.

\section{References}

[1] C. L. Wong, J. Holroyd-Leduc, and S. E. Straus, "Does this patient have a pleural effusion?" Journal of the American Medical Association, vol. 301, no. 3, pp. 309-317, 2009.

[2] M. E. Froudarakis, "Diagnostic work-up of pleural effusions," Respiration, vol. 75, no. 1, pp. 4-13, 2008.

[3] E. Diaz-Guzman and R. A. Dweik, "Diagnosis and management of pleural effusions: a practical approach," Comprehensive Therapy, vol. 33, no. 4, pp. 237-246, 2007.

[4] M. A. Rothlin, R. Naf, M. Amgwerd, D. Candinas, T. Frick, and O. Trentz, "Ultrasound in blunt abdominal and thoracic trauma," Journal of Trauma, vol. 34, no. 4, pp. 488-495, 1993.

[5] N. Xirouchaki, E. Magkanas, K. Vaporidi et al., "Lung ultrasound in critically ill patients: comparison with bedside chest radiography," Intensive Care Medicine, pp. 1-6, 2011.

[6] D. Lichtenstein, J. S. Hulot, A. Rabiller, I. Tostivint, and G. Mezière, "Feasibility and safety of ultrasound-aided thoracentesis in mechanically ventilated patients," Intensive Care Medicine, vol. 25, no. 9, pp. 955-958, 1999.

[7] K. L. Eibenberger, W. I. Dock, M. E. Ammann, R. Dorffner, M. F. Hormann, and F. Grabenwoger, "Quantification of pleural effusions: sonography versus radiography," Radiology, vol. 191, no. 3, pp. 681-684, 1994.

[8] P. C. Yang, K. T. Luh, D. B. Chang, H. D. Wu, C. J. Yu, and S. H. Kuo, "Value of sonography in determining the nature of pleural effusion: analysis of 320 cases," American Journal of Roentgenology, vol. 159, no. 1, pp. 29-33, 1992.

[9] J. Kurian, T. L. Levin, B. K. Han, B. H. Taragin, and S. Weinstein, "Comparison of ultrasound and CT in the evaluation of pneumonia complicated by parapneumonic effusion in children," American Journal of Roentgenology, vol. 193, no. 6, pp. 1648-1654, 2009.

[10] A. Peris, L. Tutino, G. Zagli et al., "The use of point-of-care bedside lung ultrasound significantly reduces the number of 
radiographs and computed tomography scans in critically ill patients," Anesthesia and Analgesia, vol. 111, no. 3, pp. 687692, 2010.

[11] D. Feller-Kopman, "Ultrasound-guided thoracentesis," Chest, vol. 129, no. 6, pp. 1709-1714, 2006.

[12] J. Turner and J. Dankoff, "Thoracic ultrasound," Emergency Medicine Clinics of North America, vol. 30, no. 2, pp. 451-473, 2012.

[13] M. Balik, P. Plasil, P. Waldauf et al., "Ultrasound estimation of volume of pleural fluid in mechanically ventilated patients," Intensive Care Medicine, vol. 32, no. 2, pp. 318-321, 2006.

[14] B. Coley, "Chest sonography in children: current indications, techniques and imaging findings," Radiologic Clinics of North America, vol. 49, no. 5, pp. 825-846, 2011.

[15] K. F. Pinotti, S. M. Ribeiro, and A. J. M. Cataneo, "Thorax ultrasound in the management of pediatric pneumonias complicated with empyema," Pediatric Surgery International, vol. 22, no. 10, pp. 775-778, 2006.

[16] F. J. H. Brims, S. M. Lansley, G. W. Waterer, and Y. C. G. Lee, "Empyema thoracis: new insights into an old disease," European Respiratory Review, vol. 19, no. 117, pp. 220-228, 2010.

[17] C. H. Chen, W. Chen, H. J. Chen et al., "Transthoracic ultrasonography in predicting the outcome of small-bore catheter drainage in empyemas or complicated parapneumonic effusions," Ultrasound in Medicine and Biology, vol. 35, no. 9, pp. 1468-1474, 2009.

[18] H. J. Chen, Y. H. Yu, C. Y. Tu et al., "Ultrasound in peripheral pulmonary air-fluid lesions: color Doppler imaging as an aid in differentiating empyema and abscess," Chest, vol. 135, no. 6, pp. 1426-1432, 2009.

[19] T. Havelock, R. Teoh, D. Laws, and F. Gleeson, "Pleural procedures and thoracic ultrasound: British Thoracic Society pleural disease guideline 2010," Thorax, vol. 65, no. 2, pp. i61i76, 2010.

[20] C. Moore, "Ultrasound-guided procedures in emergency medicine," Ultrasound Clinics, vol. 6, no. 2, pp. 277-289, 2011.

[21] S. Nicolaou, A. Talsky, K. Khashoggi, and V. Venu, "Ultrasound-guided interventional radiology in critical care," Critical Care Medicine, vol. 35, supplement 5, pp. S186-S197, 2007.

[22] D. A. Gervais, A. Petersein, M. J. Lee, P. F. Hahn, S. Saini, and P. R. Mueller, "US-guided thoracentesis: requirement for postprocedure chest radiography in patients who receive mechanical ventilation versus patients who breathe spontaneously," Radiology, vol. 204, no. 2, pp. 503-506, 1997.

[23] S. J. Koenig, M. Narasimhan, and P. H. Mayo, "Thoracic ultrasonography for the pulmonary specialist," Chest, vol. 140, no. 5, pp. 1332-1341, 2011.

[24] C. E. Daniels and J. H. Ryu, "Improving the safety of thoracentesis," Current Opinion in Pulmonary Medicine, vol. 17, no. 4, pp. 232-236, 2011.

[25] F. V. Adams and V. Galati, "M-mode ultrasonic localization of pleural effusion. Use in patients with non diagnostic physical and roentgenographic examinations," Journal of the American Medical Association, vol. 239, no. 17, pp. 1761-1764, 1978.

[26] T. W. Barnes, T. I. Morgenthaler, E. J. Olson, G. K. Hesley, P. A. Decker, and J. H. Ryu, "Sonographically guided thoracentesis and rate of pneumothorax," Journal of Clinical Ultrasound, vol. 33, no. 9, pp. 442-446, 2005.

[27] G. W. Boland, G. S. Gazelle, M. J. Girard, and P. R. Mueller, "Asymptomatic hydropneumothorax after therapeutic thoracentesis for malignant pleural effusions," American Journal of Roentgenology, vol. 170, no. 4, pp. 943-946, 1998.
[28] R. D. Brandstetter, M. Karetzky, R. Rastogi, and J. D. Lolis, "Pneumothorax after thoracentesis in chronic obstructive pulmonary disease," Heart and Lung, vol. 23, no. 1, pp. 67-70, 1994.

[29] H. G. Colt, N. Brewer, and E. Barbur, "Evaluation of patient-related and procedure-related factors contributing to pneumothorax following thoracentesis," Chest, vol. 116, no. 1, pp. 134-138, 1999.

[30] D. R. Duncan, T. I. Morgenthaler, J. H. Ryu, and C. E. Daniels, "Reducing iatrogenic risk in thoracentesis: establishing best practice via experiential training in a zero-risk environment," Chest, vol. 135, no. 5, pp. 1315-1320, 2009.

[31] D. R. Grogan, R. S. Irwin, R. Channick et al., "Complications associated with thoracentesis. A prospective, randomized study comparing three different methods," Archives of Internal Medicine, vol. 150, no. 4, pp. 873-877, 1990.

[32] H. R. Harnsberger, T. G. Lee, and D. H. Mukuno, "Rapid, inexpensive real-time directed thoracentesis," Radiology, vol. 146, no. 2, pp. 545-546, 1983.

[33] J. Heidecker, J. T. Huggins, S. A. Sahn, and P. Doelken, "Pathophysiology of pneumothorax following ultrasoundguided thoracentesis," Chest, vol. 130, no. 4, pp. 1173-1184, 2006.

[34] T. Josephson, C. A. Nordenskjold, J. Larsson, L. U. Rosenberg, and M. Kaijser, "Amount drained at ultrasound-guided thoracentesis and risk of pneumothorax," Acta Radiologica, vol. 50, no. 1, pp. 42-47, 2009.

[35] P. W. Jones, J. P. Moyers, J. T. Rogers, R. M. Rodriguez, Y. C. Gary Lee, and R. W. Light, "Ultrasound-guided thoracentesis: is it a safer method?" Chest, vol. 123, no. 2, pp. 418-423, 2003.

[36] S. J. Liang, C. Y. Tu, H. J. Chen et al., "Application of ultrasound-guided pigtail catheter for drainage of pleural effusions in the ICU," Intensive Care Medicine, vol. 35, no. 2, pp. 350-354, 2009.

[37] P. H. Mayo, H. R. Goltz, M. Tafreshi, and P. Doelken, "Safety of ultrasound-guided thoracentesis in patients receiving mechanical ventilation," Chest, vol. 125, no. 3, pp. 10591062, 2004.

[38] G. Mynarek, K. Brabrand, J. Å. Jakobsen, and A. Kolbenstvedt, "Complications following ultrasound-guided thoracocentesis," Acta Radiologica, vol. 45, no. 5, pp. 519-522, 2004.

[39] P. V. O’Moore, P. R. Mueller, and J. F. Simeone, "Sonographic guidance in diagnostic and therapeutic interventions in the pleural space," American Journal of Roentgenology, vol. 149, no. 1, pp. 1-5, 1987.

[40] K. Pihlajamaa, M. K. Bode, T. Puumalainen, A. Lehtimäki, S. Marjelund, and T. Tikkakoski, "Pneumothorax and the value of chest radiography after ultrasound-guided thoracocentesis," Acta Radiologica, vol. 45, no. 8, pp. 828-832, 2004.

[41] V. Raptopoulos, L. M. Davis, G. Lee, C. Umali, R. Lew, and R. S. Irwin, "Factors affecting the development of pneumothorax associated with thoracentesis," American Journal of Roentgenology, vol. 156, no. 5, pp. 917-920, 1991.

[42] C. E. Ravin, "Thoracocentesis of loculated pleural effusions using grey scale ultrasonic guidance," Chest, vol. 71, no. 5, pp. 666-668, 1977.

[43] C. J. Yu, P. C. Yang, D. B. Chang, and K. T. Luh, "Diagnostic and therapeutic use of chest sonography: value in critically ill patients," American Journal of Roentgenology, vol. 159, no. 4, pp. 695-701, 1992.

[44] C. E. Gordon, D. Feller-Kopman, E. M. Balk, and G. W. Smetana, "Pneumothorax following thoracentesis: a systematic review and meta-analysis," Archives of Internal Medicine, vol. 170, no. 4, pp. 332-339, 2010. 
[45] Y. H. Liu, Y. C. Lin, S. J. Liang et al., "Ultrasound-guided pigtail catheters for drainage of various pleural diseases," American Journal of Emergency Medicine, vol. 28, no. 8, pp. 915-921, 2010.

[46] G. Volpicelli, M. Elbarbary, M. Blaivas et al., "International evidence based recommendations for point of care lung ultrasound," Intensive Care Medicine, vol. 38, no. 4, pp. 577591, 2012. 


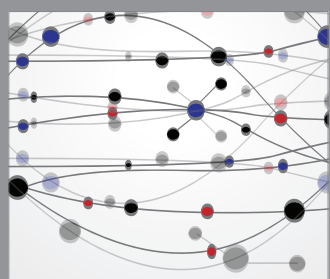

The Scientific World Journal
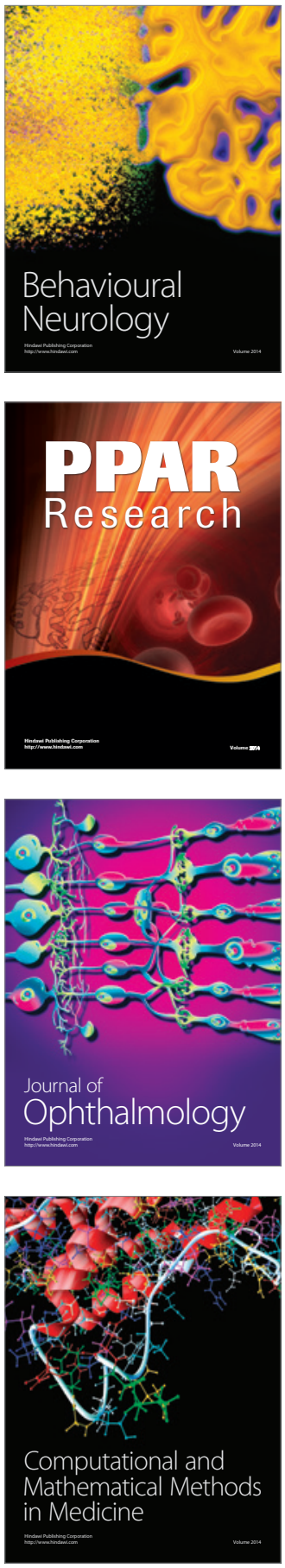

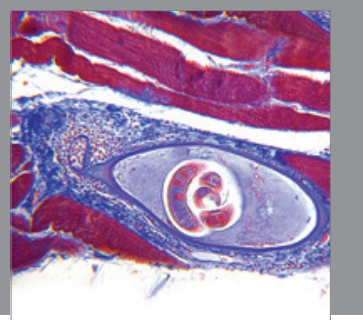

Gastroenterology

Research and Practice
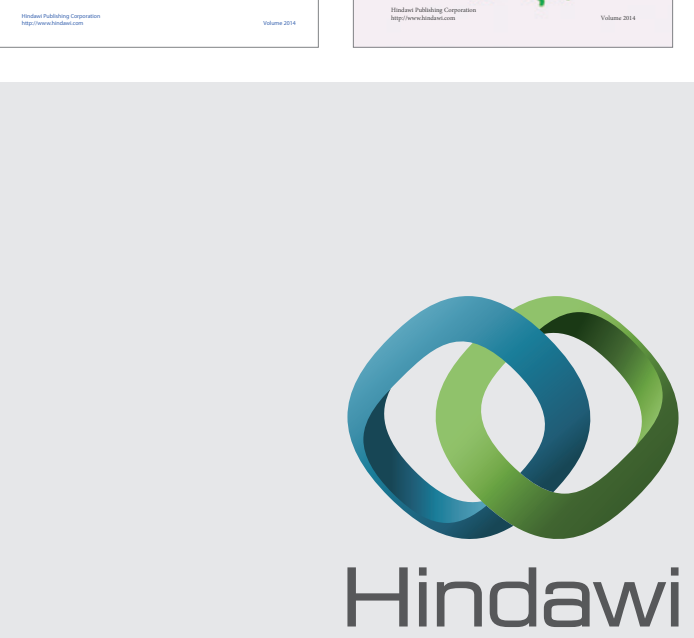

Submit your manuscripts at

http://www.hindawi.com
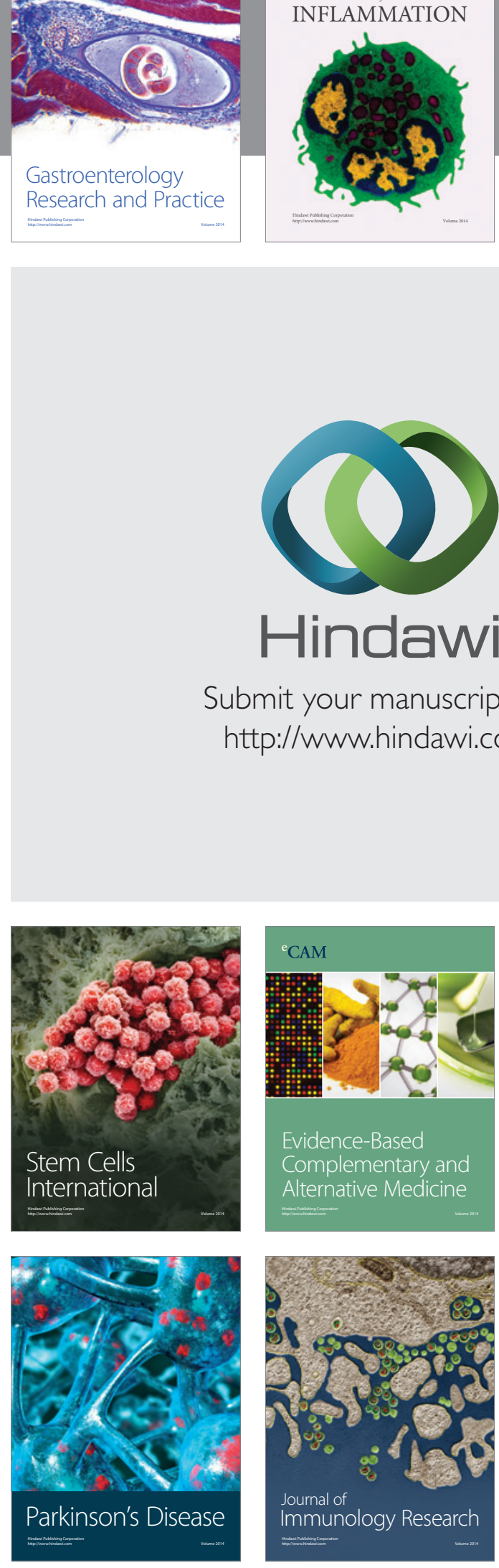

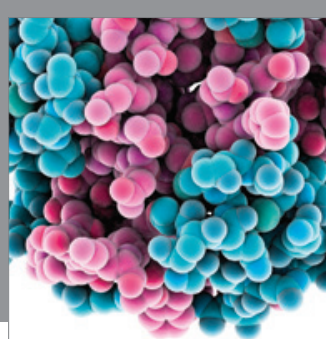

Diabetes Research
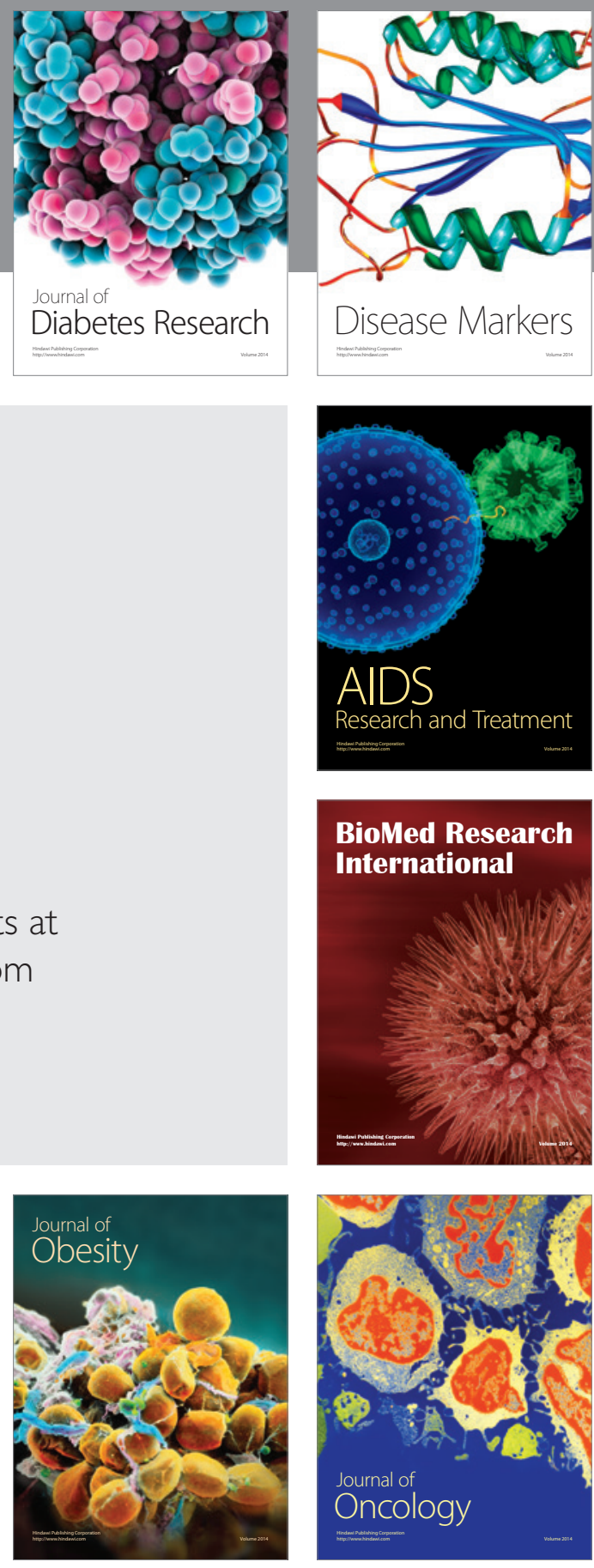

Disease Markers

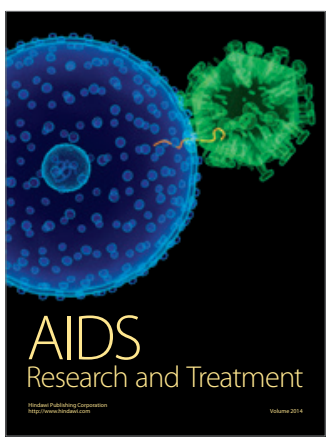

BioMed Research

International
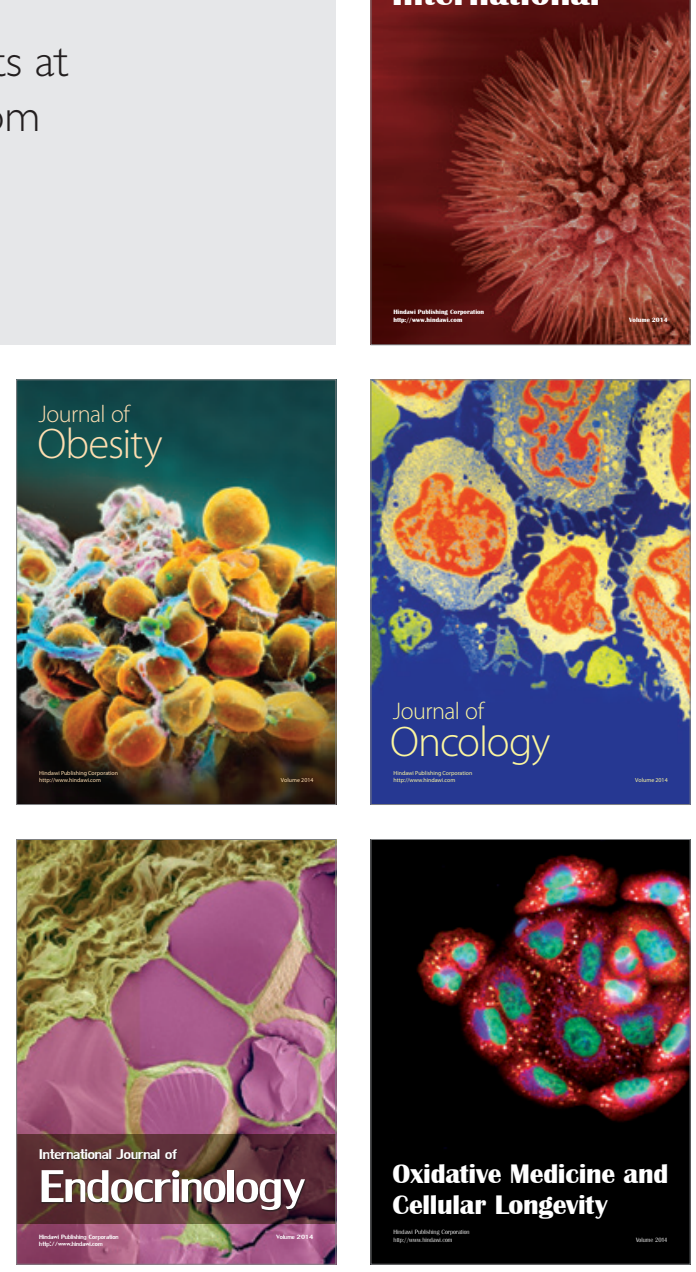